\title{
Corneal Topographic and Biomechanical Changes after Small Incision Lenticule Extraction (SMILE) In Myopic Eyes
}

\author{
Nour Elden A Abdelhalim \\ Ophthalmology Department, Faculty of Medicine, Al- Azhar University, Cairo, Egypt
}

\begin{abstract}
Background: Small incision lenticule extraction (SMILE) offers laser vision correction by using a less invasive technique that creates a lenticule inside the intact cornea. This procedure limits the biomechanical strength of the cornea and reduces flap-induced complications.

Purpose: To evaluate outcome, post-operative corneal topographic and biomechanical changes in myopic patients who had undergone SMILE.

Methods: The study included 40 eyes of 20 patients treated by SMILE for myopia and myopic astigmatism. Data included uncorrected and best corrected visual acuity (UCVA and BCVA), spherical equivalent (SE), central pachymetry Pentacam (oculus) topography to evaluate changes in keratometric readings (K1 and K2) and asphericity $(\mathrm{Q})$. Corneal hysteresis $(\mathrm{CH})$ and corneal resistance factor $(\mathrm{CRF})$ were measured with ocular response analyzer. Patients were treated and followed for 12 months.

Results: SMILE procedure for the correction of myopia and myopic astigmatism was performed on 40 eyes of 20 patients. There was highly significant difference between pre and postoperative keratometric regarding $\mathrm{K} 1$ and $\mathrm{K} 2$ was change from $42.9 \pm 0.82$ and $39.8 \pm 1.14$ to $43.9 \pm 1.21$ and $40.5 \pm 1.18$ respectively (P value $<0.001)$.Corneal resistance factor and hysteresis were also change from $11.55 \pm 1.29 \mathrm{~mm} \mathrm{Hg}$ and $11.68 \pm 1.40$ $\mathrm{mm} \mathrm{Hg}$ to $9.47 \pm 1.29 \mathrm{~mm} \mathrm{Hg}$ and $8.49 \pm 1.54 \mathrm{~mm} \mathrm{Hg}$, respectively) $(\mathrm{P}<.0001)$. At the end of follow up UDVA was $20 / 20$ or better in $86 \%$ of eyes.

Conclusions: Biomechanical stability with small-incision lenticule extraction has been demonstrated with establishment of myopic and astigmatic corrective ability, so SMILE represents a safe and effective refractive option. The incidence of intraoperative and or postoperative complications remains minimal. Although visual recovery may be slower than LASIK in most cases.
\end{abstract}

Key words: SMILE, LASIK, Myopia and Topography

\section{INTRODUCTION}

Laser in-situ keratomileusis (LASIK) is currently the most common laser refractive procedure for the treatment of myopia. Its advantages include early postoperative improvement in visual acuity and minimal postoperative patient discomfort. Although LASIK patients report $95 \%$ satisfaction, a spectrum of complicated side effects can negatively impact results ${ }^{(1)}$. The femtosecond laser has been used to cut LASIK corneal flaps with high precision for over a decade. The ability to perform these highly accurate cuts to the corneal tissue has sparked new enquiry into a method of lamellar refractive surgery that may be less invasive ${ }^{(2,3)}$.

In 2009, small incision lenticule extraction (SMILE) was approved in Europe. SMILE potentially offers more advantages than femtosecond assisted LASIK as it does not require the creation of a flap thus avoiding flap-related early or late complications. The absence of flap creation with minimal disruption of the anterior stromal architecture as the corneal lenticule is extracted from the mid stroma allows for much greater preservation of biomechanical integrity and stability of the cornea. Minimal disruption of the anterior corneal surface epithelium, Bowman's layer and anterior stroma may be associated with less risk of dry eye ${ }^{(4)}$.

Small incision lenticule extraction (SMILE) offers a paradigm shift in laser vision correction by using a less invasive technique that creates a lenticule inside the intact cornea and subsequently removing it through an incision, typically less than $4 \mathrm{~mm}$ in size ${ }^{(5)}$. The development of a procedure that limits the damage to corneal nerve fibers and preserves the biomechanical strength of the cornea reduces the 
potential for patient discomfort and flap-induced complications including incomplete and irregular flap cuts, thin flaps, buttonholes and free caps, as well as the associated risk of induced astigmatism, and dry eye ${ }^{(6)}$. Further studies e confirmed that SMILE appears to be safe, predictable and efficacious in the correction of myopia ${ }^{(7,8)}$.

The purpose of this study was to evaluate postoperative corneal topographic and biomechanical changes in patients with myopia and myopic astigmatism who had SMILE procedure.

\section{Patients and methods}

All participants were provided written informed consent that explained the details of the procedure and study protocol in accordance with the principles of the Declaration of Helsinki. Inclusion criteria were ages 18 years older, normal topography, normal anterior segment by slit-lamp microscopy, and normal fundus by dilated funduscopic examination. Exclusion criteria were glaucoma, history of ocular disease, trauma or surgery, systemic disease, pregnancy, or systemic corticosteroids use. Soft contact lenses were removed at 2 weeks and hard contact lenses at least 3 weeks before preoperative measurements. The study was approved by the Ethics Board of Al-Azhar University.

Corneal biomechanical parameters were measured using ORA preoperatively and at 1 week and 1 and 3 months postoperatively. Four successful measurements were taken and the highest waveform score was taken into analysis (Waveform Score $\geq 3.5$ ) using 16 ORA software (version 3.01; Reichert Ophthalmic Instruments).

\section{Surgical technique}

All surgeries in this study were performed by authors. The Visu Max femtosecond laser system (500 kHz; Carl Zeiss Meditec, Jena, Germany) was used. Sterile draping and insertion of the speculum after application of topical anesthesia (Tetracaine Hydrochloride $0.5 \%$,), the patient's eye was centered and docked with the curved interface cone before application of suction fixation. The laser is activated and initially cut the posterior surface of the refractive lenticule followed by creation of the lenticule border. The anterior surface of the refractive lenticule was then formed which extends beyond the posterior lenticule diameter by $0.5 \mathrm{~mm}$ to form the anterior stromal layer (ASL), and was followed by a vertical curvilinear cut to form the entrance wound. The following femtosecond laser parameters was used: $100 \mu \mathrm{m}$ ASL thickness, $7.5 \mathrm{~mm}$ anterior-plane cut diameter, $6.5 \mathrm{~mm}$ optical zone of lenticule, $160 \mathrm{~nJ}$ of energy with lenticule side-cut angles at $135^{\circ}$. A $2.1 \mathrm{~mm}$ entrance wound was created centered between 9 and 12 o'clock in all cases. The spot distance and tracking spacing was $4.5 / 4.5 \mu \mathrm{m}$ for the posterior lenticule plane, $2.5 / 2.5 \mu \mathrm{m}$ for the lenticule sidecut, $4.5 / 4.5 \mu \mathrm{m}$ for the anterior lenticule plane and 2.5/2.5 $\mu \mathrm{m}$ for the entrance wound side-cut. After the suction was released, a Sinsky hook was first used to separate the entrance wound cut made by the femtosecond laser, and then to identify the edge of the lenticule under the ASL. A Chansue Dissector (CRD) was then used to separate the posterior surface of the ASL from the anterior surface of the lenticule then to release the lenticule from its bed. The lenticule is then grasped and extracted with a pair of non-toothed serrated microforceps through the small incision.

\section{Statistical analysis}

Data were collected, revised and statistically analyzed using an IBM personal computer with Statistical Package of Social Science (SPSS) version 22 (SPSS, Inc, Chicago, Illinois, USA). The quantitative data were presented as mean, standard deviations and ranges when their distribution found parametric and qualitative data were presented as number and percentages a $\mathrm{P}$ value of $<0.05$ was considered significant statistically.

\section{Results}

The study included 40 eyes, 26 eyes of 13 female patients $(65 \%)$ and 14 eyes of 7 male patients (35\%) and age ranged from 21 to 32 years old, the mean \pm SD age was $25.26 \pm 3.72$. All patients were treated for myopia up to -9D and myopic astigmatism up to -3.5 using the SMILE procedure. 
Corneal Topographic and Biomechanical Changes after Small Incision Lenticule...

Table (1): Represent preoperative refraction and topographic parameters.

\begin{tabular}{|l|c|c|}
\hline & Range & Mean \pm SD \\
\hline Refraction & $-1.5 \mathrm{D}$ to $-9 \mathrm{D}$ & $-5.68 \pm 3.08$ \\
\hline Pachymetry & 515 to 575 & $557.60 \pm 16.60$ \\
\hline Ant. Km & 42.1 to 47.1 & $44.13 \pm 1.19$ \\
\hline Post. Km & -6.5 to -6.8 & $-6.29 \pm 0.18$ \\
\hline Rh anterior surface & 7.4 to 7.97 & $7.67 \pm 0.16$ \\
\hline Rv anterior surface & 7.38 to 8.16 & $7.66 \pm 0.26$ \\
\hline Rh posterior surface & 6.08 to 6.75 & $6.37 \pm 0.21$ \\
\hline Rv posterior surface & 6.1 to 6.83 & $6.27 \pm 0.28$ \\
\hline Ant. Q- value & -0.51 to -0.32 & $-0.38 \pm 0.09$ \\
\hline Post. Q-value & -0.52 to- 0.04 & $-0.29 \pm 0.19$ \\
\hline
\end{tabular}

Table (1). Represent preoperative refraction and topographic parameters.

All eyes had a CDVA of 20/25 or better preoperatively and $95 \%$ had a UDVA of 20/25 or better at 1 day postoperatively. UDVA remained stable, with $95 \%$ of eyes achieving 20/25 or better after 12 months.

In this study, there was highly significant difference between mean pre and postoperative Keratometric results regarding K1 and K2. It was $42.9 \pm 0.82$ and $39.8 \pm 1.14$ respectively and ranging between 41.4 - 44.6 preoperative to $38.0-41.7$ postoperative for $\mathrm{K} 1$ and $43.9 \pm 1.21$ and $40.5 \pm 1.18$ respectively and ranging between $42.1-47.1$ preoperative to $38.4-43$ postoperative for K2 ( $\mathrm{P}$ value $<0.001$ ) ( Figure 1) .

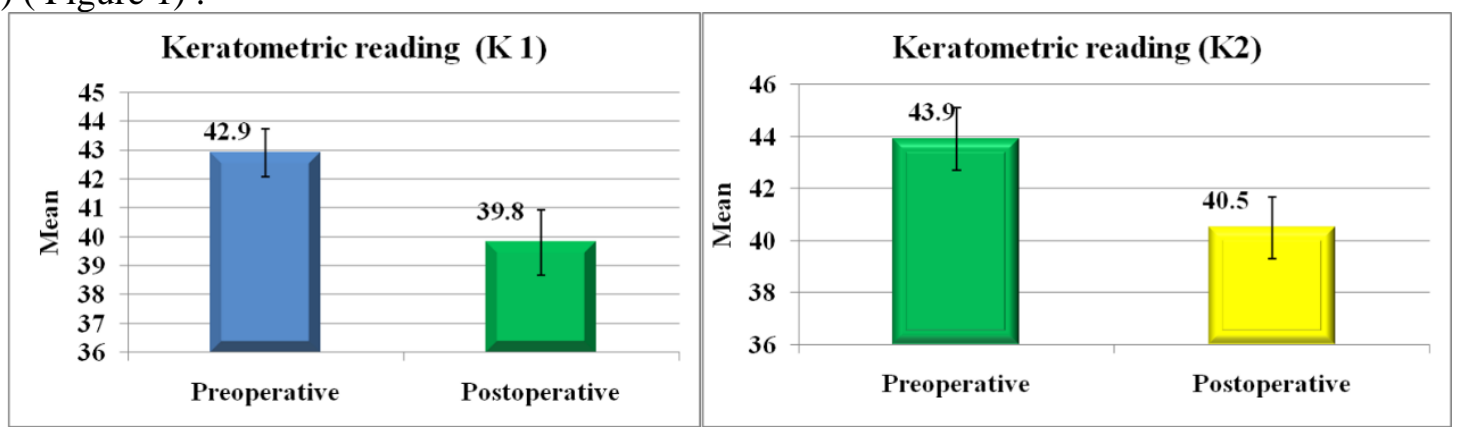

Figure (1): Pre and postoperative mean keratometry (K1 and K2).

There was a significant changes in the radii of curvature $\mathrm{Rh}$ and $\mathrm{Rv}$ for the anterior corneal surface $(P=0.001)$, but no significant change at the posterior corneal surface $(P=0.054$ and $P=0.076$, respectively).

Mathematical calculation of the anterior corneal elevation was done preoperatively at both the paracentral rings ( $4 \mathrm{~mm}$ from the center) and peripheral rings $(7 \mathrm{~mm}$ from the center) of the central $9 \mathrm{~mm}$ zone of the cornea to obtain the change in elevation. There was a significant increase in anterior corneal elevation at $4 \mathrm{~mm}$ and $7 \mathrm{~mm}$ from the center 6 months after procedure $(P$ $=0.001$ and $P=0.001$, respectively). Mathematical calculation of the posterior corneal elevation was done preoperatively at both the paracentral rings $(4 \mathrm{~mm}$ from the center) and peripheral rings ( $7 \mathrm{~mm}$ from the center) of the central $9 \mathrm{~mm}$ zone of the cornea to obtain the change in elevation. We found no significant change in posterior corneal elevation at $4 \mathrm{~mm}$ and
$7 \mathrm{~mm}$ from the center $(\mathrm{P}=0.834$ and $\mathrm{P}=0.961$, respectively) 6 months after procedure.

A significant change in Q- value for the anterior and posterior corneal surfaces was found 6 months postoperatively $(P=0.001)$. There was a tendency for the $\mathrm{Q}$ value to become more positive after myopic correction.

$\mathrm{CH}$ and $\mathrm{CRF}$ showed a significant decrease after SMILE we found a significant decrease in $\mathrm{p} 1$ and $\mathrm{p} 2$ postoperatively $(\mathrm{P}<0.05)$, also we found a stronger correlation between CRF and $\mathrm{CCT}$ than $\mathrm{CH}$ and $\mathrm{CCT}$.

There was highly significant difference between mean pre and postoperative corneal thickness it was 545.6 32.7 and $471.9 \pm 39.7$ respectively and ranged $493-586$ preoperatively to $398-531$ postoperatively ( $\mathrm{P}$ value $<0.001$ ).

There was highly significant difference between pre and postoperative mean intraocular pressure $(\mathrm{mm} \mathrm{hg})$.So there was significant decrease of mean intraocular pressure $(\mathrm{mm}$ 
hg).between pre and postoperative it was $12.7 \pm$ 1.44 and $11.1 \pm 1.41$ respectively and ranging between 10.7 - 15.6 preoperative to ranging between $8.30-13.2$ postoperative ( $\mathrm{p}$ value $<0.001)$.

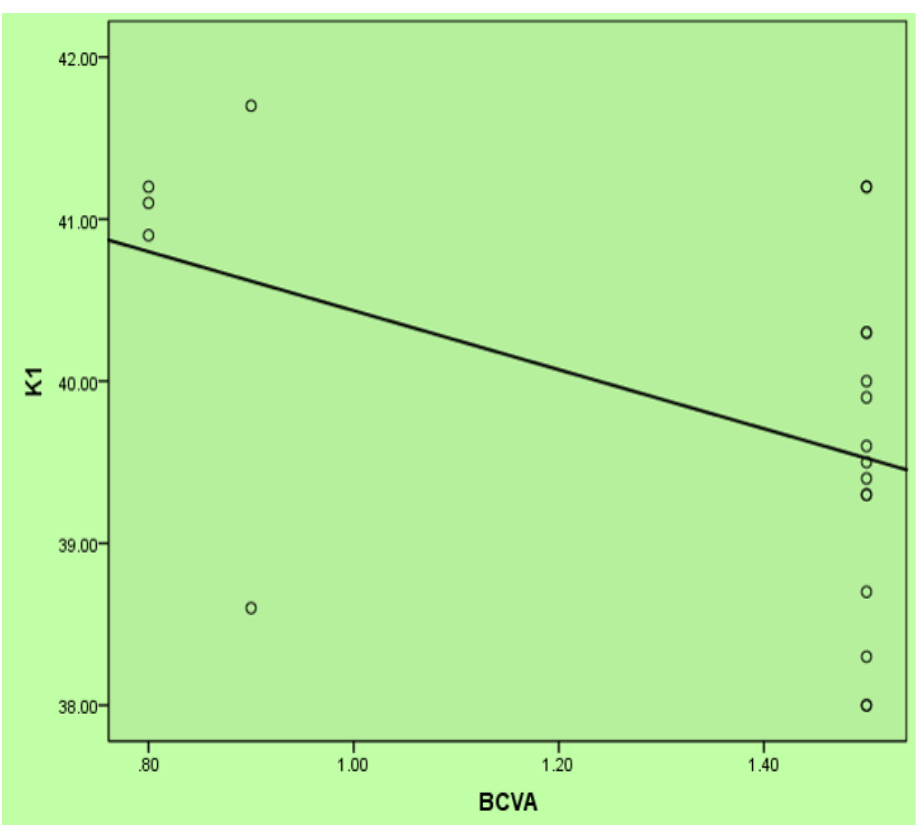

Figure (2): Correlation between postoperative BCVA and keratometry (K1).

There was negative correlation between postoperative asphericity and keratometry results (P value < 0.05 ) as shown in Figure (3).

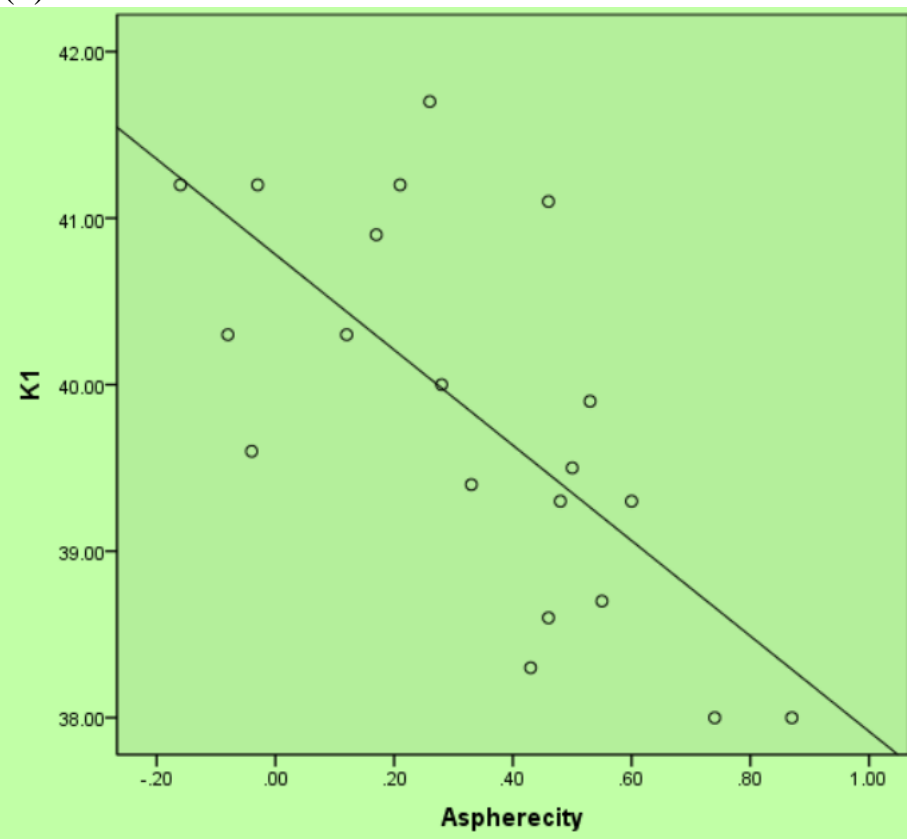

Figure (3): Correlation between postoperative aspherecity and keratometry (K1).

No significant side effects or complications were observed in any of the eyes in this study. 


\section{DISCUSSION}

This study aimed to evaluate corneal topographic and corneal biomechanical changes of small incision lenticular extraction (SMILE). Despite its relatively recent clinical introduction, small-incision lenticule extraction has become an important surgical procedure and may become a prominent component in refractive surgery modalities ${ }^{(9,10)}$.

In this study, there was highly significant difference between mean pre and postoperative keratometric results regarding $\mathrm{K} 1$ and $\mathrm{K} 2$ it was $42.9 \pm 0.82$ and $39.8 \pm 1.14$ respectively and ranging between $41.4-44.6$ preoperative to 38.0 - 41.7 postoperative (P value $<0.001)$ Anders et al. ${ }^{(11)}$ reported that there wass highly significant difference between pre and postoperative mean keratometric reading. In their study there was significant decrease of mean average keratometry between pre and postoperative it was $43.43 \pm 1.47$ and $37.98 \pm 1.49$ respectively and ranging between $40.79-46.72$ preoperative to ranging between 35.62-41.44postoperative, after 6 month of follow up of SMILE was significant .

A significant change in Q-value for the anterior and posterior corneal surfaces was found 6 months postoperatively .There was a tendency for the $\mathrm{Q}$ value to become more positive after myopic ablation.

Our study revealed a significant change in the radii of curvature $\mathrm{Rh}$ and $\mathrm{Rv}$ for the anterior corneal surface, but no significant change at the posterior corneal surface. It was possible that surgery affects corneal stability more strongly in the vertical direction. Further, mathematical calculation of the anterior corneal elevation was done preoperatively at both the paracentral rings ( $4 \mathrm{~mm}$ from the center) and peripheral rings (7 $\mathrm{mm}$ from the center) of the central $9 \mathrm{~mm}$ zone of the cornea to obtain the change in elevation. Our results show a significant increase in anterior corneal elevation at $4 \mathrm{~mm}$ and $7 \mathrm{~mm}$ from the center 3 months after LASIK.

We found no significant change in posterior corneal elevation at $4 \mathrm{~mm}$ and $7 \mathrm{~mm}$ from the center 6 months after LASIK. In 2014 Kamiya et al. ${ }^{(12)}$ found a statistically significant difference in posterior corneal elevation pre- and post-SMIL.

In current study, $\mathrm{CH}$ and $\mathrm{CRF}$ showed a significant decrease after SMILE. We found a significant decrease in $\mathrm{p} 1$ and $\mathrm{p} 2$ postoperatively $(\mathrm{P}<.05)$, which was consistent with other refractive surgery studies ${ }^{(13,15)}$. There was a stronger correlation between CRF and CCT than $\mathrm{CH}$ and CCT and it was suspect that the CRF may be intended to reflect the overall corneal rigidity, which was consistent with other studies (16,17) .When compared with the LASIK the CH, CRF, p1area, and p2area decreased less in SMILE in myopia greater than $-6.00 \mathrm{D}^{(15)}$.

In this study, there was highly significant difference between mean pre and postoperative corneal thickness it was $545.6 \pm 32.7$ and $471.9 \pm$ 39.7 respectively and ranged between $493-586$ preoperative to $398-531$ postoperative ( $\mathrm{P}$ value $<0.001)$.

Similar to our study, Han et al. ${ }^{(17)}$ reported that there is highly significant difference between pre and postoperative mean corneal thickness. So there is significant decrease of mean corneal thickness between pre and postoperative it was $544 \pm 31$ and $434 \pm 34$ respectively and ranging between 490-613 preoperative to ranging between 376-505 postoperative, after 6 month of follow up of SMILE $(\mathrm{P}<0.001)$.

Similar to our study, Han et al. ${ }^{(17)}$ reported that there was highly significant difference between pre and postoperative mean intraocular pressure $(\mathrm{mm} \mathrm{Hg})$. So there was significant decrease of mean intraocular pressure ( $\mathrm{mm} \mathrm{Hg}$ )between pre and postoperative. It was $16 \pm 3$ and $9 \pm 2$ respectively and ranging between 11-24 preoperative to ranging between 5- 13 postoperatively, after 6 month of follow up of SMILE ( $p$ value $<0.001$ ).

Glare, halos, and night vision complaints were reported by $10-15 \%$ of patients which was less than what was reported by LASIK correction 18,19. However, the single, small vertical cut used in SMILE minimizes collapse or stromal damage, inducing fewer aberrations, leading to better quality of vision.

\section{Conclusions}

Biomechanical stability with smallincision lenticule extraction has been demonstrated with establishment of myopic and astigmatic corrective ability. So SMILE represents a safe and effective refractive option. 
The incidence of intraoperative and or postoperative complications remains minimal. Although visual recovery may be slower than LASIK in most cases.

\section{References}

1. Alio JL, Muftuoglu O, Ortiz D, PérezSantonja JJ, Artola A, Ayala MJ et al. (2008): Ten-year follow-up of laser in situ keratomileusis for high myopia. Am $\mathbf{J}$ Ophthalmol., 145:46-54.

2. Slade SG (2007): The use of the femtosecond laser in the customization of corneal flaps in laser in situ keratomileusis. Curr Opin Ophthalmol., 18:314-7.

3. Kim P, Sutton GL, Rootman DS (2001): Applications of the femtosecond laser in corneal refractive surgery. Curr Opin Ophthalmol., 22:238-44.

4. McAlinden $\mathbf{C ( 2 0 1 2 ) : ~ C o r n e a l ~ r e f r a c t i v e ~}$ surgery: past to present. Clin Exp Optom., 95(4):386-98.

5. Reinstein DZ, Archer TJ, Gobbe M (2014): Small incision lenticule extraction (SMILE) history, fundamentals of a new refractive surgery technique and clinical outcomes. Eye and Vision, 1:3.

6. Sekundo W, Kunert K, Russmann C, Gille A, Bissmann W, Stobrawa G et al. (2008): First efficacy and safety study of femtosecond lenticule extraction for the correction of myopia: six-month results. J Cataract Refract Surg., 34:1513-20.

7. Shah R, Shah S, Sengupta S (2011): Results of small incision lenticule extraction: Allin-one femtosecond laser refractive surgery. J Cataract Refract Surg.; 37(1):127-37.

8. Vestergaard A, Ivarsen AR, Asp S, Hjortdal J (2012): Small-incision lenticule extraction for moderate to high myopia: Predictability, safety, and patient satisfaction. J Cataract Refract Surg., 38(11):2003-10

9. Sekundo W, Kunert KS, Blum M (2011): Small incision corneal refractive surgery using the small incision lenticule extraction (SMILE) procedure for the correction of myopia and myopic astigmatism: results of a 6 month prospective study. $\mathrm{Br} \mathrm{J}$ Ophthalmol., 95(3):335-9.

10. Moshirfar M, McCaughey MV, Reinstein DZ, Shah R, Santiago-Caban L, Fenzl CR (2015): Small-incision lenticule extraction. J Cataract Refract Surg., 41(3):652-65.

11. Anders H, Vestergaard M, Jakob G et al. (2014): Efficacy, safety, predictability, contrast sensitivity, and aberrations after femtosecond laser lenticule extraction. J Cataract Refract Surg., 40:403-411.

12. Kamiya K, Shimizu K, Igarashi A, Kobashi H (2014): Visual and Refractive Outcomes of Femtosecond Lenticule Extraction and Small-Incision Lenticule Extraction for Myopia. Am J Ophthalmol., 157(1):128-134.e2.

13. Sekundo W, Bönike $K$, Mattausch $P$, Wiegand W (2003): Six-year follow-up of laser in situ keratomileusis for moderate and extreme myopia using a first-generation excimer laser and microkeratome. J Cataract Refract Surg., 29:1152-8.

14. Solomon KD, de Castro LE F, Sandoval HP, Biber JM, Groat B, Neff KD et al. (2009): LASIK world literature review: quality of life and patient satisfaction. Ophthalmology, 116(4):691-701.

15. Dada T, Sudan R, Sinha R, Ray M, Sethi H, Vajpayee RB (2003): Results of laser in situ keratomileusis for myopia of -10 to -19 diopters with a Technolas 217 laser. J Refract Surg., 19(1):44-7.

16. Sekundo W, Gertnere J, Bertelmann T, Solomatin I (2014): One-year refractive results, contrast sensitivity, high-order aberrations and complications after myopic small-incision lenticule extraction (ReLEx SMILE) Graefes Arch Clin Exp Ophthalmol., 252(5):837-43.

17. Han $T$, Zheng $K$, Chen $Y$ et al. (2016): Four-year observation of predictability and stability of small incision lenticule extraction. BMC Ophthalmol., 16:149. 